\title{
Corticospinal System Development Depends on Motor Experience
}

\author{
John H. Martin, ${ }^{1,3}$ Michelle Choy, ${ }^{1}$ Seth Pullman, ${ }^{2}$ and Zhuo Meng ${ }^{1}$ \\ ${ }^{1}$ Center for Neurobiology and Behavior, Columbia University, ${ }^{2}$ Department of Neurology, Columbia University, and ${ }^{3}$ New York State Psychiatric Institute, \\ New York, New York 10032
}

\begin{abstract}
Early motor experiences have been shown to be important for the development of motor skills in humans and animals. However, little is known about the role of motor experience in motor system development. In this study, we address the question of whether early motor experience is important in shaping the development of the corticospinal (CS) tract. We prevented limb use by the intramuscular injection of botulinum toxin A into selected forelimb muscles to produce muscle paralysis during the period of development of CS connection specificity, which is between postnatal weeks 3 and 7. CS terminations were examined using an anterograde tracer. Preventing normal forelimb use during CS axon development produced defective development of CS terminations at week 8 and in maturity. There were reductions in the topographic distribution of axon terminals, in terminal and preterminal branching, and in varicosity density. This suggests that limb use is needed to refine CS terminals into topographically specific clusters of dense terminal branches and varicosities. To determine correlated effects on motor behavior, cats were tested in a prehension task, to reach and grasp a piece of food from a narrow food well, when the neuromuscular blockade dissipated (by week 10) and in maturity (week 16). Preventing normal limb use also produced a prehension deficit later in development and in maturity, in which there was a loss of the supination component of grasping. This component of prehension in the cat depends on CS projections from the paw representation of rostral motor cortex to the cervical enlargement. Our findings show that motor experiences are necessary for normal development of CS terminations and function.
\end{abstract}

Key words: motor cortex; corticospinal system; spinal cord; development; postnatal, cat; prehension; botulinum toxin A

\section{Introduction}

Early motor experiences have been shown to be important in the development of motor skills in humans and animals (Zelazo et al., 1972; Solomons and Solomons, 1975; Super, 1976; Westerga and Gramsbergen, 1993; Walton, 1998). However, surprisingly little is known about the role of motor experience in motor systems development. The question of how experience shapes motor systems development is complex, but can be made simpler by examining a particular motor pathway and a motor behavior controlled primarily by that pathway. In this study, we determined whether early motor experience is important in corticospinal (CS) tract development in the cat. This pathway is critically involved in complex and adaptive movements (Porter and Lemon, 1993). We examined a component of prehension that, in the cat, depends on CS projections from motor cortex to the cervical enlargement (Alstermark et al., 1981; Martin and Ghez, 1993).

Normally, CS axons grow into the cervical cord during late prenatal and early postnatal development, and terminate within

\footnotetext{
Received 0ct. 13, 2003; revised Jan. 5, 2004; accepted Jan. 6, 2004.

This work was supported by National Institutes of Health Grant NS33835 and by the March of Dimes Birth Defects Foundation. We thank XiuLi Wu for histology and help during experiments, Dr. M. Osman and G. Asfaw for veterinary care, and Allergan Inc. for the gift of most of the botulinum toxin A used in this study.

Correspondence should be addressed to Dr. John H. Martin, Center for Neurobiology and Behavior, Columbia University, 1051 Riverside Drive, New York, NY 10032. E-mail: jm17@columbia.edu. D0I:10.1523/JNEUROSCI.4616-03.2004

Copyright $\odot 2004$ Society for Neuroscience $\quad$ 0270-6474/04/242122-11\$15.00/0
}

the gray matter soon thereafter (Wise et al., 1977; Stanfield, 1992; Eyre et al., 2000). By weeks 2-3 CS terminations within the spinal gray matter in cats are locally sparse but topographically extensive, with branches extending to regions not normally contacted in maturity (Li and Martin, 2001, 2002). Subsequently, terminations are refined to a mature topography by week 7 (Li and Martin, 2000) by activity-dependent competition (Martin and Lee, 1999; Martin et al., 1999). The movement repertoire of kittens expands during this period (Villablanca and Olmstead, 1979; Levine et al., 1980), and by the end, cats are weaned and become dependent on their motor skills for catching prey.

We propose that the production of limb movements during the period of the development of connectional specificity is essential for the normal maturation of the CS system. As in human infants, in whom arm movements are expressed soon after birth (Bower et al., 1970; Hofsten, 1982; Meer et al., 1995), kittens also produce a wide range of limb movements in the context of nursing, locomotion, and play (Levine et al., 1980). Without these movements, the developing CS system, and other motor circuits, might not be able to orchestrate normal CS axon refinement.

To prevent limb use, we blocked neuromuscular transmission with intramuscular botulinum toxin injections in selected forelimb muscles. This method does not impede movement of other body parts and does not interfere with interactions with the mother and littermates. Neuromuscular blockade produced by botulinum toxin reverses rapidly in the kitten, allowing us to examine skilled limb use after the effects dissipated. We show that 
Table 1. List of cases and procedures

\begin{tabular}{clllll}
\hline Cat & $\begin{array}{l}\text { BTX-start } \\
\text { reach, weeks }\end{array}$ & $\begin{array}{l}\text { Post-BTX } \\
\text { experience } \\
\text { period }\end{array}$ & $\begin{array}{l}\text { Start } \\
\text { reach, } \\
\text { weeks }\end{array}$ & $\begin{array}{l}\text { Stop } \\
\text { reach, } \\
\text { weeks }\end{array}$ & $\begin{array}{l}\text { Age when } \\
\text { killed, weeks }\end{array}$ \\
\hline BTX & & & & & \\
1 & 4 & 8 & 10 & 12 & 13 \\
2 & 4 & 8 & 10 & 12 & 13 \\
3 & & & & & 8 \\
4 & & & & & 8 \\
$5^{a}$ & 10 & 14 & 16 & 20 & 30 \\
6 & 10 & 14 & 16 & 20 & 33 \\
7 & 10 & 14 & 16 & 20 & 77 \\
Control & & & & & 8 \\
1 & & & & & 8 \\
2 & & & & & $>1$ year \\
$3^{a}$ & & & & & \\
4 & & & & & \\
5 & & & & & \\
\end{tabular}

${ }^{a}$ One animal received unilateral intramuscular BTX injections, but bilateral motor cortex tracer injections. Behaviora data from the BTX-injected side, and corresponding spinal tissue, correspond to case 5. Data from the noninjected side correspond to control case 3 .

preventing forelimb use during the development of CS connections produced persistent reductions in the topographic distribution of axon terminations, in segmental axon branches, and in varicosity density. These anatomical changes correlated with a prehension impairment. Our findings show, for the first time, that motor experiences are necessary for the normal development of CS terminations.

\section{Materials and Methods}

We used 12 cats in this study (see Results and Table 1). Our basic paradigm was to prevent the use of one limb during the CS axon terminal refinement period (weeks 3-7) with neuromuscular blockade produced by botulinum toxin (termed BTX-injected limb). CS axon terminals were examined using an anterograde tracer either at the end of the refinement period (week 8) or in maturity. The morphology of terminals controlling the nonused side (i.e., ipsilateral to the side of BTX injections but contralateral to their origin in cortex) was compared with that of controls. Correlated changes in motor performance were examined in selected animals later in development (week 12) or in maturity (week 20). We compared the performance of the BTX-injected limb with that of the noninjected (control) limb.

Botulinum toxin injections. All cats used in this study were obtained from a supplier accredited by the Association for Assessment and Accreditation of Laboratory Animal Care International. All experiments were conducted with the approval of the New York State Psychiatric Institute and Columbia University institutional animal care and use committees. Forelimb use was prevented by the intramuscular injection of botulinum toxin A (BTX; Allergan, Irvine, CA). BTX is a clostridial neurotoxin that acts inside nerves and blocks neurotransmitter release via their proteolytic activity directed on the SNARE protein [soluble $\mathrm{N}$-ethylmaleimide-sensitive factor attachment protein (SNAP) receptor] SNAP-25 (Schiavo et al., 2000). After injection into muscle, the toxin is taken up by the motoneuron axon terminal, where it acts to block acetylcholine release. In our preliminary experiments we determined that 15 $\mathrm{U}$ injected directly into the belly of forelimb muscles was sufficient to prevent limb use for at least 1 week in young kittens (see Results). This amount is similar to what is injected into human intrinsic hand and small forearm muscles for treating focal dystonias, but the duration is much shorter (Pullman et al., 1996). During preliminary experiments we determined that injections into the elbow flexors and extensors, together with the wrist plantar-flexor compartment, were sufficient for preventing limb use. The shoulder and wrist extensor muscle compartments were not injected. Having several muscle groups intact offered the opportunity to determine whether the animals contracted these intact mus- cles during testing. As we describe below, this appears not to have been the case because animals neglected use of the limb.

BTX was diluted to a final concentration of $100 \mathrm{U} / \mathrm{ml}$ of saline just before injection. The skin over the ventral forearm and arm was shaved to permit palpating the muscles to be injected. Separate injections (15 U BTX/150 $\mu$ l sterile saline per muscle) were made directly into the large plantar flexor belly on the ventral forearm, into the elbow flexor compartment, and two separate injections into the triceps (from its lateral and medial aspects). Animals were returned to their home cage after the injections.

We determined the efficacy of the injections using the following behavioral tests to evoke limb movements: contact and proprioceptive placing reactions, withdrawal response to moderate (but not noxious) paw pressure, and assessment of elbow strength. Within $2 \mathrm{~d}$ after the initial injection, contact and proprioceptive placing reactions were essentially absent. Kittens normally attempt to withdraw the paw when it is held or lightly squeezed; this reaction was not present $2 \mathrm{~d}$ after BTX injection. To assess strength at the elbow, the animal was supported under the chest and the hindlimbs were raised off the testing surface. While standing only on one or the other forelimb, pressure was applied to the shoulder of the supporting limb. The control limb would support considerable downward force before the elbow yielded and flexed. In contrast, the injected limb not only would yield at the onset of force testing but commonly did not have sufficient strength to support the animal's forequarter against the force of gravity alone.

We reinjected the same muscles at weekly intervals to prevent the muscles from regaining strength because, in the kitten, the time course of BTX actions in limb muscles is less than in the adult cat (Misiaszek and Pearson, 2002). After the fourth injection at week 6 , animals regained elbow strength and limb responsivity within $\sim 2$ weeks. Behavioral testing began either 4 or 10 weeks after the last injection. As we report in Results, after recovery from the blockade (i.e., during prehension training and testing) animals did not avoid using the injected limb. They routinely used either limb (see training description below), and showed no signs of weakness in lifting the paw to the height of the target and inserting the paw into the target tube. Recording electromyographic (EMG) activity confirmed the efficacy of the blockade and subsequent recovery (see Results and Fig. 1). EMG activity was recorded using nichrome wires that were de-insulated at the tips and inserted via 25 gauge hypodermic needles directly into the belly of the muscle. The lowfrequency filter cutoff was set at $10 \mathrm{~Hz}$ and the high-frequency cutoff at $10 \mathrm{kHz}$; the gain was 1000. EMG activity was acquired using a National Instruments (Austin, TX) digitizer at a sampling rate of $10 \mathrm{kHz}$.

Prehension training. The method of training was the same for all animals. First, the animals were allowed to use either limb to reach to grasp a piece of food ( $5 \mathrm{~mm}$ cube of beef) from a platform held in front of an opening in the cage. The height of the platform was $5.5 \mathrm{~cm}$ above the cage floor, which was the starting height of the paw. Next, the animals were required to grasp the food morsel from within a cylindrical tube $(3.2 \mathrm{~cm}$ internal diameter; $5.0 \mathrm{~cm}$ deep; Martin and Ghez, 1993; Martin et al., 1995). Initially the food was placed close to the opening of the tube; gradually it was moved to the back of the tube, thereby forcing the animal to reach deep into the tube. Finally, the session was divided into separate blocks for testing the control and BTX-injected limbs. To prevent the use of one limb during testing, we covered the paw with a bootie or tape. Animals were trained for 1 week and tested for 1 week $(n=2)$ or 3 weeks $(n=3)$. Testing sessions were videotaped, and data were analyzed from the last three sessions. For each reach during testing sessions we scored: (1) entrance into the cylindrical target well without contacting its walls, and (2) the presence of forearm supination during food withdrawal (see supplementary video, available at www.jneurosci.org). We focused on these two prehension characteristics because aiming and grasping errors characteristically increase with CS system dysfunction in cats (Alstermark et al., 1981; Martin and Ghez, 1993). Statistical analyses were conducted using the program StatView (SAS Institute, Cary, NC) for the Macintosh computer (Apple Computers, Cupertino, CA).

Surgical procedures and tracer injections. Animals were administered atropine $(0.04 \mathrm{mg} / \mathrm{kg}$, i.m. $)$ and a broad-spectrum antibiotic (Combiotic; $20,000 \mathrm{U} / \mathrm{kg}$, i.m.). During surgery, the body temperature was main- 
tained at $39^{\circ} \mathrm{C}$ by a heating pad. Animals were tranquilized with diazepam $(0.1 \mathrm{mg} / \mathrm{kg}$, i.m.). Anesthesia, to maintain an areflexive condition, was induced using a mixture of ketamine hydrochloride $(30 \mathrm{mg} / \mathrm{kg}$, i.m. and acepromazine $(0.03 \mathrm{mg} / \mathrm{kg}$, i.m.). A tracheal tube was inserted, and anesthesia was maintained with isoflurane. Animals of each age group received buprenorphine $(0.03 \mathrm{mg} / \mathrm{kg}$, i.m.) for analgesia after surgery.

Animals were mounted in a stereotaxic head holder (David Kopf Instruments, Tujunga, CA). Under aseptic conditions, a craniotomy was made over the lateral portion of the frontal lobe contralateral to the BTX-injected limb to expose the forelimb representation of motor cortex (Chakrabarty and Martin, 2000). The dura was incised over the area in which tracer was to be injected. We used the anterograde tracer biotinylated dextran amine (BDA; Molecular Probes, Eugene, OR). The pipette containing BDA (5\% BDA in PBS, pH 8.0) was lowered to a depth of $1-1.5 \mathrm{~mm}$, depending on the age of the animal. Injections were made within the lateral portion of the forelimb representation in the anterior and posterior sigmoid gyri. After a 5 min waiting period, we performed ionophoresis on tracer $(7 \mu \mathrm{A} ; 7 \mathrm{sec}$ on; $7 \mathrm{sec}$ off; $20 \mathrm{~min}$; pipette tip diameter, $\leq 20 \mu \mathrm{m}$ ). After the injection was complete, we waited $5 \mathrm{~min}$ before removing the pipette.

Histology. After an appropriate survival period [2 weeks for postnatal week 6 (PW6) animals, killed at 8 weeks; 4 weeks for older animals], animals were deeply anesthetized (Nembutal, $30 \mathrm{mg} / \mathrm{kg}$, i.v.) and perfused transcardially with warm saline $\left(39^{\circ} \mathrm{C} ; 200-400 \mathrm{ml}\right.$ for $2 \mathrm{~min}$, depending on body weight). Heparin was injected (200-500 U, i.v.) at the onset of the saline perfusion. This was followed by $4 \%$ paraformaldehyde in $0.1 \mathrm{M}$ phosphate buffer (PB; pH 7.3-7.4 at $4^{\circ} \mathrm{C} ; 1000 \mathrm{ml} / \mathrm{kg}$ body weight), infused initially at the rate of $100-200 \mathrm{ml} / \mathrm{min}$ and then adjusted to a slower rate to produce a total perfusion time of 20-30 min. The brain and spinal cord were removed, postfixed in the same fixative at $4^{\circ} \mathrm{C}$ for $2 \mathrm{hr}$, and then transferred to $20 \%$ sucrose in PB overnight, which was sufficient for the tissue to sink to the bottom of the container. Frozen sections $(40 \mu \mathrm{m})$ through the C7-T1 spinal cord (64-96 transverse sections, collected in serial order), and parasagittal sections through cortex were cut and processed. Sections were collected in $0.1 \mathrm{M} \mathrm{PB}$ and rinsed three times ( 5 min for each) in $0.1 \mathrm{M}$ PBS, pH 7.4. Sections were incubated in PBS containing 1\% avidin-biotin complex reagent (Vector Laboratories, Burlingame, CA) and $0.2 \%$ Triton $\mathrm{X}-100$ for $2 \mathrm{hr}$ at room temperature. After rinsing twice in $0.1 \mathrm{M}$ PBS and once in $0.01 \mathrm{M}$ Tris-buffered saline (TBS, pH 7.6), the tissue was incubated with the chromogen diaminobenzidine (DAB) for 6-30 min in a solution containing $1 \mathrm{DAB}$ tablet (10 mg; Sigma-Aldrich, St. Louis, $\mathrm{MO}$ ) and $12 \mathrm{ml}_{\text {of }} \mathrm{H}_{2} \mathrm{O}_{2}$ in $15 \mathrm{ml}$ of $0.01 \mathrm{~m}$ TBS. After one additional rinse in TBS and two in PBS, sections were mounted on gelatin-coated slides, air-dried overnight, dehydrated, and coverslipped.

Morphological analyses. We conducted three analyses on the anatomical data. In the first, which was a topographic analysis of the distribution of label in the gray matter, we used a camera lucida to trace all label on two adjacent sections for each case. For each section, we digitized the tracings and, using the skeletonize function in NIH Image, we computed the total length of label present on each slide. This function erodes all lines (i.e., traced axons) to a single pixel width. The total number of pixels thus corresponds to the total length of axonal label on the section. The total pixel number was calibrated according to a length measurement, which yielded the total length of labeled CS axons in micrometers.

In the second analysis, which was a morphometric analysis of CS axon terminals, we traced all label present within a rectangular field $(648 \times 512$ $\mu \mathrm{m})$ at $400 \times$ magnification within the region of densest terminations on two adjacent slides using a camera lucida. For each axon fragment traced, we measured the length, number of branch points, and number of axon varicosities. This is similar to one of our earlier studies on normal CS axon terminal development ( $\mathrm{Li}$ and Martin, 2001).

In the third analysis, we used single axon reconstruction on serial sections, as in our previous study (Li and Martin, 2002). This approach also has been used to characterize the development of retinogeniculate projections in kittens (Antonini and Stryker, 1993). All reconstructions were made using a camera lucida. Sections for reconstructing terminals were chosen according to two criteria: (1) optimal density of labeling, and (2) the absence of artifacts. It should be noted that our ability to reconstruct axons across serial transverse sections could be achieved because we labeled only a small number of cortical neurons with small tracer injections and used high-resolution differential interference contrast microscopy.

For each section analyzed, BDA-labeled axons were drawn at $100 \times$ magnification, including the collateral branch, main trunk branches in the gray matter, and many of the smaller branches. Between three and five serial sections were drawn in this manner. Using a light box, the tracings were aligned by drawn landmarks, and we identified axons to be reconstructed on the basis of close contiguity of the cut ends of the axons across sections. In this way, we were able to reconstruct all collateral branches terminating in the set of serial sections analyzed.

Next, we traced, at $400 \times$ magnification, only those axons identified as candidates for reconstruction on the low-power tracings. Segmental axon terminals were reconstructed from the collateral branch in the lateral white matter, medially into the gray matter, and then to the most distal branches. When we encountered the end of an axon branch without a terminal varicosity, we drew surrounding landmarks (neuronal profiles, blood vessels, etc.), and then continued to reconstruct the axon on the adjoining rostral or caudal section. We used the continuity of these local landmarks to guide tracing on the subsequent sections. Varicosities were defined, as in our previous study (Li and Martin, 2001), as axonal swellings that were at least three times wider and darker staining than adjoining portions of the axon. For each varicosity, we focused through the section to verify that the varicosity was planar and not a bend or kink in the axon. For each reconstructed axon, we counted the number of axonal branches and varicosities and measured total axon length (as above) to calculate the density of axonal varicosities (i.e., number of varicosities/ $\mu \mathrm{m}$ of axon). Statistical analyses were conducted using the program StatView (SPS Institute, Cary, NC) for the Macintosh computer.

\section{Results}

\section{Experimental paradigm and animal database}

Twelve cats were used for this study ( $n=7$ BTX; $n=5$ control) (Table 1). We prevented the use of one limb with neuromuscular blockade produced by intramuscular injections of BTX. For assessing the motor effects of preventing limb use, we compared the performance of the BTX-injected (test) and noninjected (control) limbs. Therefore, each animal served as its own control. We used a combination of age-matched controls and bilateral motor cortex tracer injection for assessing the effects of preventing limb use on CS axon terminal development.

The clinical and experimental literature indicates that the neuromuscular blockade produced by intramuscular BTX injection is temporary and reversible (Jankovic, 1994; Pullman et al., 1996). In PW3 animals, the target muscle groups were each injected with $15 \mathrm{U}$ of BTX (see Materials and Methods). Within 24 $\mathrm{hr}$, there was a reduction in strength of the injected limb. After $2 \mathrm{~d}$, the animal favored the limb by not using it during support or feeding and dragging it during locomotion. The injected muscles also felt flaccid, and the limb offered no resistance to passive joint movements, compared with the noninjected limb.

Control experiments showed that animals began to regain limb use 1-2 weeks after the BTX injection. They would attempt to withdraw the injected limb in response to tugging the forepaw, and there was increased resistance to passive elbow flexion and extension. On the basis of this assessment, we decided to inject BTX weekly. Our strategy was to minimize limb use by ensuring continuity of the neuromuscular blockade. The efficacy of the second and subsequent BTX injections was the same as the first. Figure 1 shows an example of EMG recordings made in an animal during neuromuscular blockade $2 \mathrm{~d}$ after BTX injection into the left triceps (top left trace) and 3 weeks later with no intervening BTX injections (top right trace). For all recordings, the animal was anesthetized with ketamine $(25 \mathrm{mg} / \mathrm{kg}$, i.m.). In our experi- 


\section{A. During left triceps BTX block}

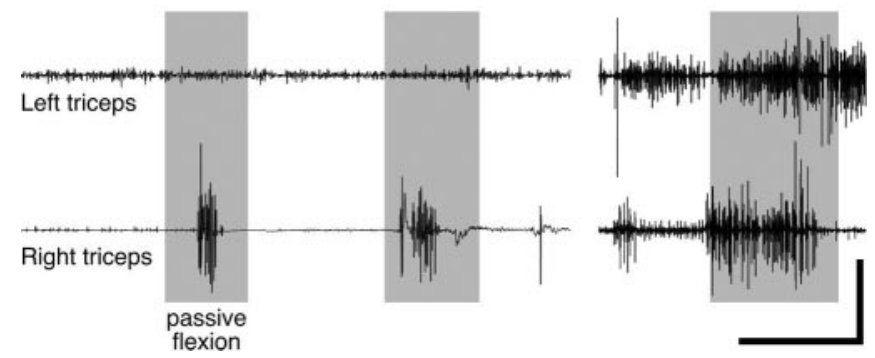

Figure 1. Effect of BTX on stretch-evoked EMG activity in the triceps muscle. A, Recording made during neuromuscular blockade $2 \mathrm{~d}$ after intramuscular BTX injection into the left triceps ( $15 \mathrm{U}$ into the medial compartment and $15 \mathrm{U}$ lateral). The gray rectangles correspond to when the right and left elbows were passively flexed. B, Recording made 3 weeks after BTX injection, after the animal regained control. Right and left EMGs were recorded concurrently during each session. Calibration: $A, 1500 \mathrm{msec}$, top, $0.025 \mathrm{~V}$; $A$, bottom, $0.1 \mathrm{~V} ; B, 500 \mathrm{msec}, 0.1 \mathrm{~V}$.

ence, ketamine anesthesia maintains muscle tone (Chakrabarty and Martin, 2000). At each time point symmetrical EMG electrode placements were made on the BTX-injected and noninjected sides. The elbow of the BTX-injected and noninjected limbs was passively flexed in tandem to approximately the same extent. Two days after BTX injection into the left biceps, passive elbow flexion did not evoke phasic EMG activity in that muscle. In contrast, there was a clear, phasic burst of triceps activity on the noninjected side during each period of passive elbow flexion. Three weeks later, passive elbow flexion evoked robust responses bilaterally. As expected, these findings show that BTX reversibly blocked stimulus-evoked EMG activity in kittens.

We determined whether there were any direct effects of repeated BTX injection on neurons within the lateral motor nuclei. We counted the number of neurons in the lateral motor nuclei (identified on dark field by medial axon bundling at the lamina 7-9 border) on the BTX-injected and noninjected sides at the eighth cervical segment (C8) (see Materials and Methods). Several studies have shown that motoneurons innervating the biceps, triceps, and wrist flexors are located at C8, as well as at adjoining rostral and caudal segments (Sterling and Kuypers, 1967; Fritz et al., 1986a,b). For each animal that received BTX injections we counted neurons on three sections separated by 200 $\mu \mathrm{m}$. Every profile was counted because the $200 \mu \mathrm{m}$ separation ensured that we would not count one cell twice. Although most of the neurons counted would have been motoneurons, we cannot exclude counting interneurons within the motor nuclei. Our counts were made on the same set of sections we used for measuring CS axon terminations. The mean number of neurons on the injected side ( $n=5$ animals) was $23.5 \pm 2.08 \mathrm{SE}$, compared with $25.1 \pm 1.98$ on the noninjected side. This difference was not significant (paired $t$ test; $t=-0.92 ; p=0.373$ ) and shows that BTX did not affect lateral motor nuclei cell counts. This shows that there were no long-term toxic effects of BTX injections.

\section{Preventing limb use between weeks 3 and 7 impairs prehension later in development and in maturity}

To determine the motor consequences of preventing early postnatal limb use, we allowed animals to recover from the neuromuscular block and trained them to perform a prehension task (see Materials and Methods). We began to train animals either at PW10, which was 4 weeks after the last BTX injection and 2-3 weeks after resumption of limb use, or week 16 , which was 10 weeks after the last BTX injection and 14 weeks after resumption of limb use (Table 1). Animals reached from within their cage into a narrow cylinder located outside the cage door to grasp and retrieve a food reward (see Materials and Methods).

Animals did not show a preference for using the BTX-injected or noninjected limbs. With either limb, the paw entered the cylinder accurately and the paw was placed on the piece of food. There were no differences in the percent of trials in which the tube was contacted on entering. This indicates that there were no differences in trajectory error (e.g., horizontal and vertical paw location at time of target entrance) because the width of the tube was approximately that of the paw. We noticed an impairment in the withdrawal phase of prehension. Video clips (in slowmotion) are presented in the supplementary material (available at www.jneurosci.org). Figure 2 shows video still frames of the withdrawal phase from a behavioral trial for the noninjected (control) limb $(A)$ and the BTX-injected (test) limb (B). Normally, animals grasp and retrieve the food reward by a coordinated digit flexion, wrist plantar flexion, and forearm supination (Alstermark et al., 1981; Martin and Ghez, 1993; Boczek-Funcke et al., 1998), although not all components of the grasp/retrieval synergy are apparent in the photos. This behavior effectively scoops food into the paw. Supination appears to be necessary to ensure that the food is held securely. Otherwise, the food is dropped after the paw leaves the tube. Figure $2 \mathrm{~A} 1$ corresponds to the frame in which food contact was made and the paw is outlined. Figure $2 A 2$ is six frames later, in which the paw begins to supinate as it is withdrawn. To facilitate comparison, the outline of the paw in Figure $2 A 1$ (white line), in which the limb is not supinated (i.e., pronated), is superimposed on the outlines in $A 2$ and $A 3$ (dashed lines), in which the limb is supinated. Because of forearm supination, the white outline of the pronated paw in the first frame differs from the dashed outlines of the second and third. Figure $2 A 3$ shows the supinated position of the paw as it leaves the cylinder. A similar set of video frames is shown in Figure $2 B$ for the BTX-injected limb (i.e., not used between weeks 3 and 7). The principal difference is that supination was absent: the paw maintains a pronated posture and merely slid from the tube, raking the food out. Note that the white and dashed outlines superimpose. Food withdrawal during the majority of trials was without supination. Data from the noninjected and BTX-injected limbs of each animal ( $n=5$ animals; three sessions each; 10 trials per session) were compared. Using stop-action, we determined whether or not supination occurred on each trial. As shown in the video clips and still frames, when supination is absent, the paw is held horizontally (i.e., $0^{\circ}$ with respect to horizontal) during withdrawal; and when present, it rotates to a nearly vertical orientation (i.e., $90^{\circ}$ ). For supination to be present, the paw must have rotated by $45^{\circ}$ or more. However, as shown in the still frames and video clips, when supination did not occur, the food was raked from the tube. The phenotypes of the withdrawal with or without supination are distinct. The rate of supination dropped from $72.8 \pm 3.9 \%$ of the trials for the noninjected side to $14.9 \pm 2.2 \%$ for the BTX-injected side (paired $t$ test; $p<0.0001$ ). In addition to the marked difference between the noninjected and BTXinjected limbs, two points are important to note. First, the noninjected limb was not supinated during every trial while retrieving the food reward. This indicates that the supination component of prehension is not obligatory. It is produced on the majority of trials, probably because it is an effective strategy for minimizing food drop (Alstermark et al., 1981; Sakamoto et al., 1989). Second, the BTX-injected limb supinated occasionally. This shows that the behavior is not absent because of muscle paralysis or atrophy. 
Two of the animals were tested between 4 and 6 weeks after the last BTX injection and, on average, reaches made by the noninjected limb were supinated $62 \%$ more often than reaches made by the BTXinjected limb. The other three animals were tested between 10 and 14 weeks after the last injection, and the average difference between percent supination for the noninjected and BTX-injected limbs was $57 \%$. Although the impairment was slightly greater when the animals had less experience to use their limb after the period of nonuse, the impairment is still robust in maturity (i.e., 16 weeks). Our results show that preventing limb use during weeks 3-7, which correspond to the CS axon terminal topographic refinement period, produced a permanent impairment in the capacity to coordinate supination with shoulder retraction during food withdrawal.

\section{Preventing limb use between weeks 3 and 7 disrupts development of the topography of the CS projection}

To determine the effects of preventing limb use on CS axon terminal development, we made small injections of the anterograde tracer BDA into motor cortex and mapped the spinal terminations. Because the topography of spinal terminations could differ slightly from one motor cortex site to another, we used two approaches for determining the effects of limb disuse on CS projections. First, we matched the location of motor cortex injection sites in pairs of injected and noninjected age-matched animals. For example, because projections from caudal and rostral motor cortex have a dorsal and ventral spinal bias, respectively (Li and Martin, 2000), we needed to compare animals with similar motor cortex injections to verify that differences in the CS terminations were caused by changes in limb use, not to the motor cortex tracer injection site. Second, after verifying that the differences were not caused by the injection site, we pooled data from animals with different motor cortex injection sites, but within a given age-matched group ( 8 weeks or mature), because comparisons between pairs of animals would not be expected to have the same statistical power as for a larger group. For all of the findings discussed below, the differences in the means for the paired-case comparisons were similar in direction and magnitude to the differences we found for the grouped data. Therefore, we present only the quantitative group data. All morphological analyses were made on CS axon terminals at C8. However, similar effects were noted for the rostral cervical cord (C3-C4; see below).

Figure 3 shows tracings of scanned parasagittal sections through the sensory motor cortex of test and control animals (or right and left cortex for one animal; see below). Tracer injection sites are indicated in gray (dark for rostral, light for caudal motor cortex). The inset is a micrograph through the injection to the right (outlined by box). Animals deprived of the use of one limb between weeks 3 and 7 showed a persistent change in the topography of CS terminations compared with controls. This is shown in Figure 4, which presents camera lucida drawings of labeling on representative sections through $\mathrm{C} 8$. CS axons in normal animals terminate densely in the medial portions of the deep laminas of the dorsal horn and intermediate zone (in addition to other sites). The functional significance of this medial pattern is not well understood, but is likely related to the presence of spinal premotor interneurons, cerebellar projection neurons, or interneurons in the area receiving monosynaptic projections from group 1a primary afferent fibers (Matsushita et al., 1979; Brown, 1981; Hongo et al., 1989). This region also receives dense CS terminations in the monkey (Armand et al., 1997). Figure $4 A 1$ shows all terminations on two adjacent sections in an 8-week-old control animal, and $4 A 2$, in a mature animal. In the young animal, terminations are densest medially but do extend more laterally. Although large single motor cortex tracer injections or multiple tracer injections produce this pattern of CS terminations, very small tracer injections may produce more restrictive patterns. In this study, injections labeled between 60 and 226 axons (both in controls and BTX). We have seen more focal patterns when injections label very few axons (less than $\sim 50$; unpublished observations).

In contrast, CS terminations in animals deprived of limb use between weeks 3 and 7 were sparse medially and dense laterally (Fig. 4B1,B2). For the young animal, CS terminals failed to be established in the medial dorsal horn and intermediate zone. Rather, dense terminals were present in the dorsolateral gray matter. Note that for the mature case in Figure 4, tracer was injected into homotopic portions of the right and left motor cortex (Table 1; case 5 BTX, case 3 control; spinal gray matter in Fig. $4 A 2$ and $4 B 2$ are both shown as if on the right side). We verified that bilateral terminations were not present in this animal (apart from the occasional axon) in two ways. First, in the case shown there were no more than a few decussating axons over the extent of the spinal cord examined (either $1 \mathrm{~cm}$ length sectioned horizontally or $5 \mathrm{~mm}$ sectioned transversely). Note that there are no labeled axons in lamina 10 (Fig. 4), where they course before 


\section{A. Non-injected B. BTX-injected}

PW 8
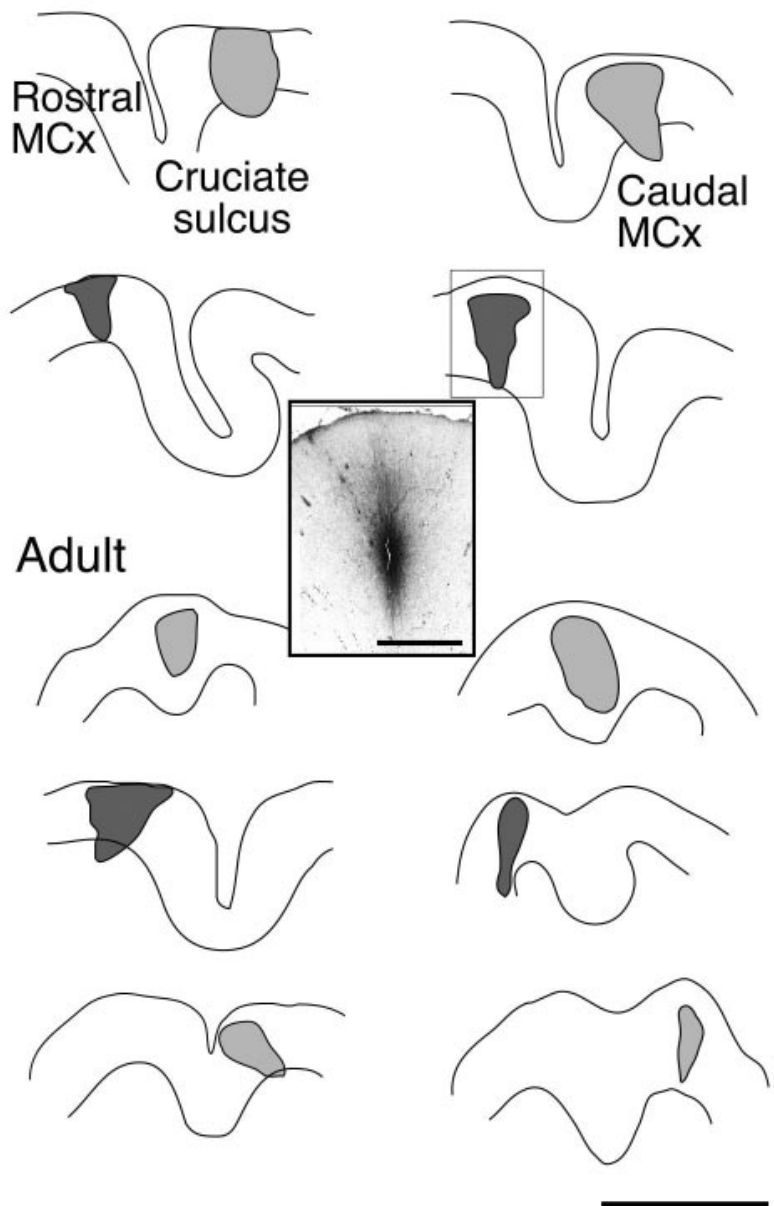

Figure 3. Motor cortex tracer injection sites. Tracings of parasagittal sections through the injection site in the motor cortex for each animal. A shows injection sites in controls or contralateral to noninjected limbs. $B$ shows injection sites contralateral to injected limbs. The micrograph inset corresponds to the injection in the second row of $B$. Injections into caudal motor cortex are light gray; injections into rostral motor cortex are dark gray. Injection border drawn to enclose all labeled neuronal cell bodies and apical and basal dendrites. Scale bar, $4 \mathrm{~mm}$; inset, $1 \mathrm{~mm}$.

decussating. Second, in two other cases studied with unilateral BDA injections (Table 1, cases 6 and 7) there were essentially no decussating axons in the cord and no more than two or three axons in the ipsilateral lateral white matter. This is comparable with previous findings (Satomi et al., 1988). The mediolateral distinction was consistent across all animals. The topographic changes we saw after BTX injections (absence or reduced terminations in the medial dorsal horn and intermediate zone) were also present in the rostral cervical cord at C3-C4. Because motoneurons of paralyzed muscles are located in the cervical enlargement (Sterling and Kuypers, 1967; Fritz et al., 1986a,b), the similarity with $\mathrm{C} 3-\mathrm{C} 4$ suggests that these anatomical effects were caused by forelimb disuse, and not paralysis of specific muscles.

For each animal, we measured the total length of labeled CS axons from the camera lucida tracings on two C8 sections using an imaging program (see Materials and Methods). The amount of labeling that was located within the geometrical medial or lateral halves of the gray matter was expressed as a percentage of total. For control PW8 animals, $65.5 \pm 3.9 \%$ of labeled CS axons

\section{A. Non-injected B. BTX-injected}
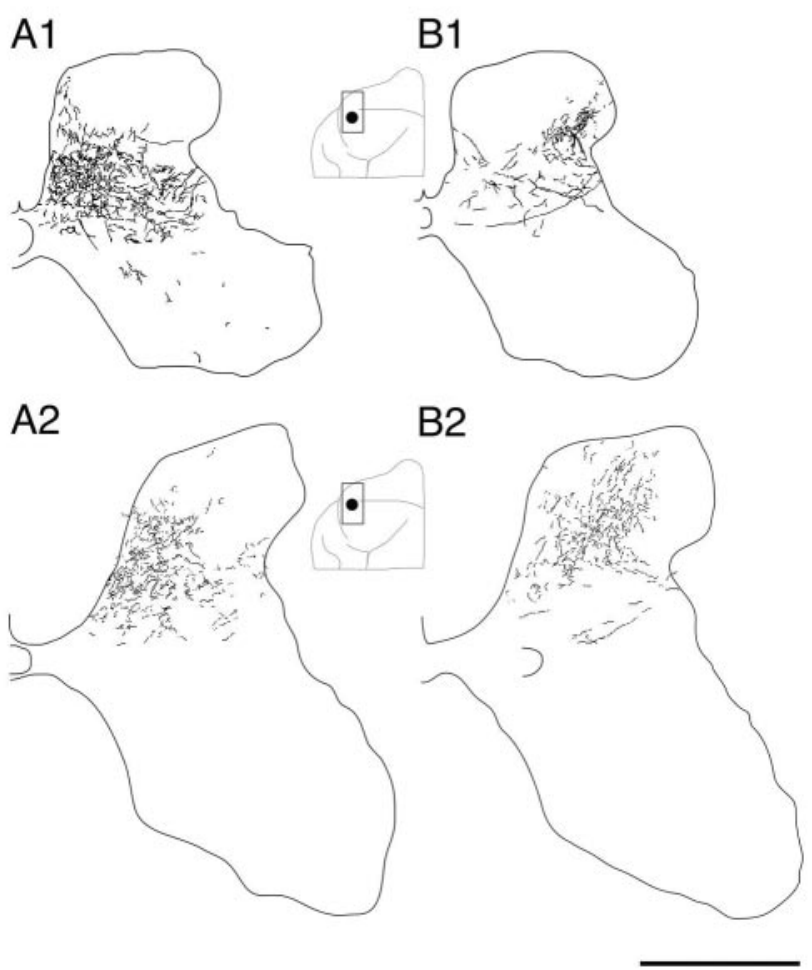

Figure 4. Camera lucida drawings of the total amount of $\mathrm{CS}$ axonal label on two adjacent $\mathrm{C8}$ sections for 4 representative cases. $A$, Control cases; 8 -week-old animal ( $A 1)$ and mature animal (A2). B, Cases in which limb use was prevented between weeks 3 and 7; 8-week-old animal (B1) and mature animal ( $B 2$ ). Insets show the locations of the injection sites, matched for $A 1$ and $B 1$ and for $A 2$ and $B 2$. Note that $B 1$ and $B 2$ are from the same animal (see Materials and Methods). Scale bar, $500 \mu \mathrm{m}$.

were located medially, compared with $28.7 \pm 3.7 \%$ in BTXinjected animals. For control adults (or for the noninjected side), $72.5 \pm 4.1 \%$ of labeled CS axons were located medially, compared with $45.8 \pm 3.3 \%$ in BTX-injected animals (or the BTXinjected side). These differences in the distribution of label were significant for both age groups $(p=0.0005)$. These findings show that preventing early postnatal limb use produced a change in the pattern of CS terminations immediately after the topographic refinement period, at week 8 , and in maturity. These findings suggest that early postnatal limb use is required for the growth and maintenance of CS connections to particular spinal gray matter fields.

\section{CS axon terminal morphology is impaired after early postnatal limb disuse}

The tracings in Figure 4 show more long and unbranched CS axons terminating on the BTX-injected side, especially at 8 weeks, than in controls. Figure 5, which presents micrographs from representative control and BTX-injected animals at $\mathrm{C} 8$, shows that there were fewer preterminal and terminal CS axons branches and varicosities when animals did not use their limb between weeks 3 and 8. Normal CS terminals are shown in Figure 5, A (8 week) and $B$ (mature). There is a single principal terminal in Figure 5, $A$ and $B$, each with several major and minor branches. For both ages, the density of varicosities and terminal branches is highest near the central portion of each terminal. In contrast, the BTX-injected animals have long, relatively unbranched, axon ter- 
minals (Fig. 5C-E), with few varicosities. All varicosities are marked with arrows in Figure $5 C-E$ to distinguish axon varicosities as putative presynaptic sites from axon kinks and other irregularities that become apparent when the contrast of the image is increased for viewing. (Note that there were too many varicosities in Fig. $5 A, B$ to mark each clearly.) At 8 weeks (Fig. 5C,D), the terminals look surprisingly similar to those soon after they have grown into the gray matter (Li and Martin, 2002), as if they failed to develop dense clusters of short branches. There are only a few varicosities on each of the axons in these figures. All of these axons were located within lamina 7, which is a principal CS terminal field. The terminals in Figure $5 E$ (three separate terminals, each indicated by an asterisk) are from a mature animal that received early postnatal BTX injections. The principal terminal in Figure $5 E$ contains more varicosities than either $5 C$ or $5 D$; however, the density is lower and the terminal branches are sparse and long compared with normals $(B)$. A similar decrease in terminal branching and varicosity density was present at $\mathrm{C} 3-\mathrm{C} 4$.

We conducted a morphometric analysis on terminal and preterminal CS axons at C8 using two approaches, one based on all labeling within a $550 \times 430 \mu \mathrm{m}$ region (which corresponded to the field of view at $400 \times$ ) within the densest field of terminations, and the second based on individual segmental branches reconstructed across several serial sections. Data from the first analysis are presented in Figure 6. Representative camera lucida tracings used for the analyses in Figure $6 A-C$ are shown in Figure $6, D$ and $E$, which are from the BTX-injected $(D)$ and noninjected $(E)$ sides of a 30-week-old animal. After BTX injections, the mean numbers of CS axon branches (Fig. $6 A$ ), mean number of varicosities (Fig. $6 B$ ), and mean varicosity density (Fig. $6 C$ ) were all significantly reduced, both at 8 weeks and in maturity (all $p<0.0001$ ). Remarkably, branch number, varicosity number, and varicosity density increased substantially (and significantly) from 8 weeks to maturity in controls (all $p<0.002$ or greater; vertical arrows, with asterisks) but hardly at all in the animals deprived of limb use between weeks 3 and 7 (small space between pairs of vertical arrows). The small increase in varicosity number for the deprived animals was not significant ( 0.118 increase; $p=0.204)$; the small increases in branch number and varicosity density were significant (branch number, 0.148 increase, $p=0.09$; varicosity density, 0.006 increase, $p=0.04$ ). These findings show that preventing limb use between weeks 3 and 7 permanently impaired the development of CS terminations. Surprisingly, during the weeks after the blocking effects of BTX dissipate, CS terminations developed no further.

To determine changes in CS axon terminal morphology at the single-cell level, we used serial transverse sections to reconstruct individual segmental branches. This technique is identical to that used in our earlier study (Li and Martin, 2002; see Materials and
Methods). Figure 7 shows reconstructed axons from representative BTX-injected and noninjected cases at C8. Varicosities are indicated by the large dots. At 8 weeks, there was extensive dorsoventral branching without clusters of short terminal branches and varicosities (Fig. 7A1, left axon). This is an immature branching pattern, resembling those seen at 3 weeks (Li and Martin, 2002), both because there are few terminal branches and varicosities and because preterminal axons extend across several laminas. In addition, the axon at the right in Figure $7 A 1$ is anomalous, both because it had several acute bends and because it also extends across several laminas. In contrast, controls at this age showed a predominantly laminar termination pattern, with dense clusters of terminal axon branches and varicosities (Fig. 7B1). In mature animals months after early postnatal limb disuse, CS terminals on the BTX-injected side still had few branches and varicosities. One axon (Fig. 7A2, top left) had a sparse terminal cluster and the other (bottom left) had anomalous dorsoventral branches.

At 8 weeks, morphometric values for the reconstructed axons from BTX-injected animals were a fraction of the control values: the branch number was reduced to $68 \%$ of control values (17 \pm 3.1 branches per axon for BTX vs $25 \pm 3$ branches/axon for controls); the varicosity number was reduced to $55.5 \%$ of controls (15.3 \pm 2.6 varicosities/axon for BTX vs $27.5 \pm 3.7$ varicos- 

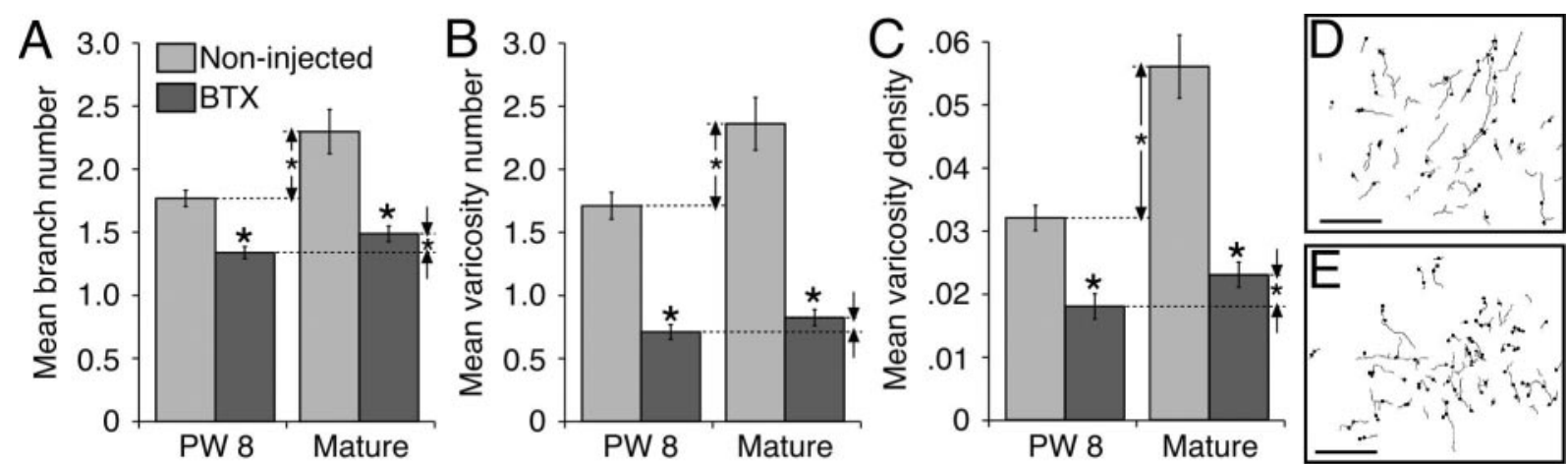

Figure 6. Effect of not using on limb between PW3 and PW7 on CS axon branch number $(A)$, varicosity number $(B)$, and varicosity density $(C)$ at $(8$. Differences between control animals at 8 weeks and maturity are shown by the double-ended arrows. Differences between BTX-injected animals at 8 weeks and maturity are shown by the size of the gap between the two single-ended arrows in each panel. D, Representative image from one of the mature animals (PW30) that received a unilateral BTX injection from which analysis was made on CS axon branches and varicosities. Gray lines indicate the axon fragments traced, and dots correspond to sites of varicosities. E, Same as D except for contralateral spinal cord (i.e., control side). Scale bars, $100 \mu \mathrm{m}$.

\section{A. BTX-injected \\ B. Non-injected}

\section{A1. 8 weeks}

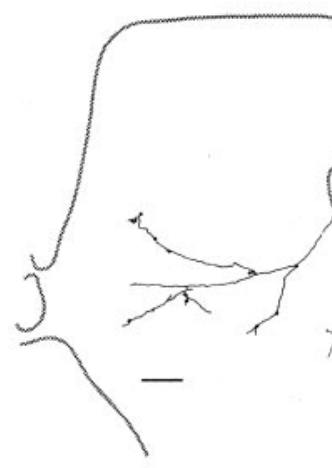

A2. Mature

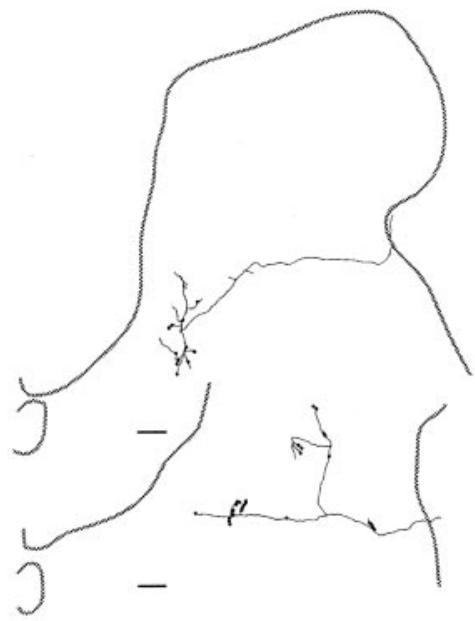

\section{B1. 8 weeks}

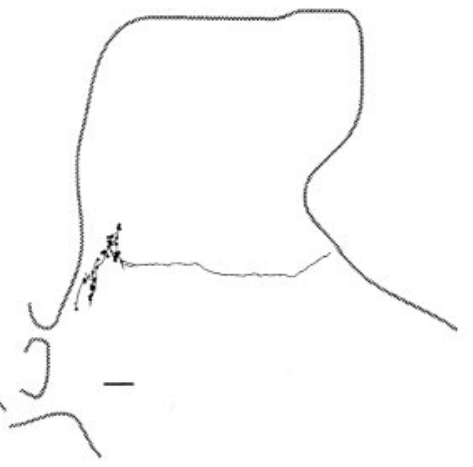

B2. Mature

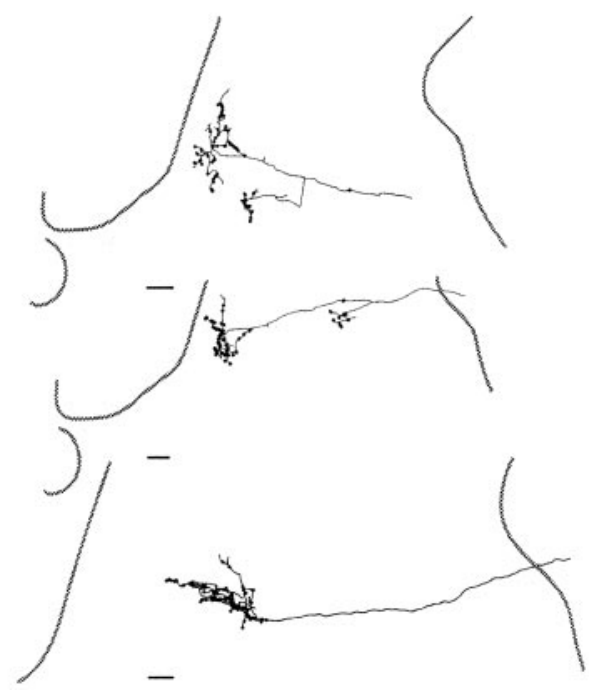

Figure 7. CS axon terminals reconstructed across several serial adjacent $C 8$ sections. $A, 8$ weeks. $B$, Mature. Two examples of axons from a BTX-injected animal are shown in $A 1$ and an age-matched control in B1. Examples of C $S$ terminals in two BTX-injected animals are shown in A2 (top, PW30; bottom, PW77), and three controls in B2 (all PW30). Scale bars, $100 \mu \mathrm{m}$.

was reduced to $47.2 \%$ of control values (13.9 \pm 1.92 branches/axon for BTX vs $29.3 \pm 3.8$ branches/axon for controls); the varicosity number was reduced to $36.9 \%$ of controls (19.4 \pm 1.9 varicosities/axon for BTX vs for $52.7 \pm 5.8$ varicosities/axon for controls); and the varicosity density was reduced to $61.7 \%(0.0145 \pm 0.0024$ varicosities/ $\mu \mathrm{m}$ axon length for BTX vs $0.0235 \pm 0.0006$ varicosities/ $\mu \mathrm{m}$ axon length for controls). Data from reconstructed CS axons parallel those from the labeled field analysis (Fig. 6) showing permanent reductions in key morphometric values. Importantly, these reductions correlate with the prehension impairment.

\section{Discussion}

Preventing forelimb use during the CS axon terminal refinement period produced a persistent abnormal topography and morphology of CS axon terminals and a prehension defect. These findings indicate the importance of normal motor experience during early postnatal life for CS system development. In addition to providing new information about how the CS system develops connections with spinal neurons, our findings have important clinical significance on the effects of restricting limb use as a therapy to treat hemiplegia in children with cerebral palsy (Willis et al., 2002). Our findings suggest that preventing movement of the normal arm (by casting, as is done in this therapy) during early development could permanently impair control of that limb. It should be noted that our use of BTX is fundamentally different from current clinical usage to treat spasticity in children and adults. We inject BTX

ities/axon for controls); and varicosity density was reduced to $43.8 \%$ ( $0.0083 \pm 0.0007$ varicosities/ $\mu \mathrm{m}$ axon length for BTX vs $0.019 \pm 0.006$ varicosities/ $\mu \mathrm{m}$ axon length for controls). In mature animals, there were similar reductions: the branch number into normal muscle in normal kittens to restrict limb use. In human patients, BTX is injected into affected muscles, where it has been shown to be effective in reducing spasticity and improving limb control (Simpson, 2000). 
Although somatic sensory signals were not blocked, the absence of limb movement would be expected to reduce the level or pattern of afferent input to the cervical cord. BTX could also affect efferent control of the muscle spindle. The somatic sensory consequences of movement and the movement itself are inextricably linked: Preventing limb movement alters somatic sensory input, and the loss of sensory input profoundly alters motor control (Ghez et al., 1990). The direction of CS axon terminal changes (reduced topography, branching, and varicosity number) is similar to but more modest than what we see after blocking neural activity in sensory-motor cortex (Friel and Martin, 2003), suggesting the importance of reduced CS descending drive. Similarly, reduced vestibulospinal tract drive may impair motoneuron development. Weightlessness during space flight influences motoneuron dendrite development (Inglis et al., 2000), suggesting that a reduction in vestibulospinal signals produced the effect.

Blocking muscle contraction also uncouples feedforward control signals with afferent feedback as a consequence of movement. This could result in increased motor system activity. However, this is unlikely because neuromuscular transmission at wrist extensor and shoulder muscles was not blocked, yet animals did not move these joints. They neglected the entire limb as if all muscle contraction were blocked.

\section{CS changes after preventing limb use could reflect absence of activity-dependent growth and enhanced axon terminal elimination}

By week 8, three key changes normally occur in the topographic pattern and morphology of CS terminations, and preventing limb use had varying influences on these developmental events. First, normally there are dense terminations in the medial dorsal horn and intermediate zone; limb disuse resulted in decreased termination density here. This late growth, which is reminiscent of delayed CS growth to motor nuclei in the monkey (Kuypers, 1962; Armand et al., 1997), may be activity dependent and could underlie the expression of skillful and adaptive limb use (Galea and Darian-Smith, 1995; Armand et al., 1997). Second, the local density of short axon terminal branches normally is increased throughout the termination field, together with an increase in axon varicosities. Limb disuse profoundly impaired this developmental process. Both failure to establish connections in particular regions and lack of axon branch and varicosity development could be caused by reduced activity-dependent growth. We were surprised that the third key developmental change, elimination of transient segmental branches, was least affected by the lack of limb use. This minimal effect may be attributable to the timing of disuse in relation to activity-dependent axon growth. Transient CS terminations are sparse at first, appear to grow as the system develops, and then are eliminated. (Theriault and Tatton, 1989; Alisky et al., 1992; Martin et al., 1999; Martin and Lee, 1999; Li and Martin, 2000). Had preventing limb use occurred later, after more extensive transient termination growth, the reduction in branch elimination might have been more noticeable. This could explain an earlier finding in neonatal rats of reduced elimination of CS neurons after limb fixation (Huttenlocher and Bonnier, 1991). Transient contralateral CS terminations are dense in the rat (Gribnau et al., 1986; Jootsten et al., 1987) and may be a more sensitive indicator of CS developmental changes. In addition, limb disuse could produce an overall reduction in activity of motor systems controlling the BTX-injected limb, including the CS system, resulting in reduced synaptic activity. This would reduce the level of activity-dependent competition and impede branch elimination (Goodman and Shatz, 1993). Thus, preventing limb use could reduce activity-dependent growth, which would lead to decreased axon branching and varicosity density, and mute synaptic competition, which would maintain branches. Together, these two changes would produce CS terminals that mimic features of the immature state (long, sparsely branched axons), suggesting an arrest of CS axon terminal development.

\section{CS system is unable to regain normal morphology and function when limb movement returns}

Once limb use was regained after the neuromuscular blocking effects of BTX dissipated there was minimal subsequent CS axon terminal growth. This is surprising because CS axons normally continue to develop denser clusters of terminals and varicosities between weeks 8 and 12. In fact, this growth can be as much as growth between weeks 3 and 8 (Li and Martin, 2001, 2002). Why then was there no recouping of lost branches and presynaptic sites after the effects of neuromuscular blockade wore off? This could reflect the long-term consequences of the competitive disadvantage produced by the anatomical changes we showed at 8 weeks. When limb use returned, other motor pathways and afferent inputs would regain normal activity because they develop prenatally (Martin et al., 1980; Ozaki and Snider, 1997) and thus would not be expected to be permanently changed by limb disuse. At each subsequent stage of development, the CS system would be at a competitive disadvantage (Martin and Lee, 1999; Eyre et al., 2001) compared with other pathways. With the existence of extensive parallel processing, other motor pathways could assume a greater role in moment-to-moment limb control. Thus, it is as if the motor cortex were driven into dysfunction by timing limb disuse with a particular CS development phase, becoming less effective in driving spinal circuits. This is expected for a developing system unable to compete with other spinal afferents.

\section{The spinal cord is an important site for organizing CS control signals for limb control}

Although the need for motor (or sensory-motor) experience for maturation of normal motor behavior has been well documented in humans and animals (Held and Bauer, 1967; Zelazo et al., 1972; Hein, 1974; Solomons and Solomons, 1975; Super, 1976; Westerga and Gramsbergen, 1993; Walton, 1998), this is the first study to correlate a persistent impairment in motor skill with impairment of motor pathway development. Our developmental findings are consistent with results in adult animals. Lesion of the CS projection to the cervical enlargement interrupts the capacity of the cats to retrieve a food reward effectively from a narrow food well, without disrupting trajectory control (Alstermark et al., 1981). Inactivation of the rostral forepaw representation in the motor cortex also disrupts supination during prehension (but with other motor impairments; Martin and Ghez, 1993), suggesting that this is the origin of the key CS projection. Thus, coordination of supination with other features of prehension shows a dominant cortical control, and parallel brain stem control is weak because in adults there is no compensation after CS lesion. We propose that the motor defect is caused by disrupted development of these CS projections. We do not, however, rule out contributions by other levels of the neuraxis. For example, BTX treatment in adult humans with dystonia has been shown to alter the topographic features of the somatic sensory cortical representation (Candia et al., 2003). Nevertheless, sparse CS axon branching and fewer varicosities would decrease the potential for control signals to activate spinal interneurons strongly. This is a likely condition for disrupting cortical motor control.

Forelimb supination during food retrieval was not abolished with limb disuse, but rather it was no longer a characteristic fea- 
ture of prehension. Because it was expressed, albeit infrequently, the circuits producing supination must be intact. However, the circuits are no longer accessed as part of an effective motor synergy during prehension. An intriguing explanation for preservation of the motor synergy but loss of context-specific access is that supination during food withdrawal is a motor primitive (Bizzi et al., 1991, 2000) produced by spinal networks (Tresch et al., 1999). Forearm supination is relatively simple in its biomechanical action, and it could be organized by spinal segmental circuits. But coordination of supination with the other components of prehension (with a particular sequence, timing, and context) needs supraspinal systems for proper execution. Our findings point to the importance of early motor experience for the CS system to learn to stitch together various movement fragments into a particular complex coordinated and purposeful movement. The anatomical changes show that the spinal cord could be an important site for development of this motor function.

\section{References}

Alisky JM, Swink TD, Tolbert DL (1992) The postnatal spatial and temporal development of corticospinal projections in cats. Exp Brain Res 88:265-276.

Alstermark B, Lundberg A, Norrsell U, Sybirska E (1981) Integration in the descending motor pathways controlling the forelimb in the cat. 9. Differential behavioural defects after spinal cord lesion interrupting defined pathways from higher centers to motoneurons. Exp Brain Res 42:299-318.

Antonini A, Stryker MP (1993) Development of individual geniculocortical arbors in cat striate cortex and effects of binocular impulse blockade. J Neurosci 13:3549-3573.

Armand J, Olivier E, Edgley SA, Lemon RN (1997) Postnatal development of corticospinal projections from motor cortex to the cervical enlargement in the macaque monkey. J Neurosci 17:251-266.

Bizzi E, Mussa-Ivaldi FA, Giszter S (1991) Computations underlying the execution of movement: a biological perspective. Science 253:287-291.

Bizzi E, Tresch MC, Saltiel P, d'Avella A (2000) New perspectives on spinal motor systems. Nat Rev Neurosci 1:101-108.

Boczek-Funcke A, Kuhtz-Buschbeck JP, Raethjen J, Paschmeyer B, Illert M (1998) Shaping of the cat paw for food taking and object manipulation: an X-ray analysis. Eur J Neurosci 10:3885-3897.

Bower TGR, Broughton JM, Moore MK (1970) Demonstration of intention in the reaching behavior of neonate humans. Nature 228:679-681.

Brown AG (1981) The organization of the spinal cord: the anatomy and physiology of identified neurones. New York: Springer.

Candia V, Wienbruch C, Elbert T, Rockstroh B, Ray W (2003) Effective behavioral treatment of focal hand dystonia in musicians alters somatosensory cortical organization. Proc Natl Acad Sci USA 100:7942-7946.

Chakrabarty S, Martin JH (2000) Postnatal development of the motor representation in primary motor cortex. J Neurophysiol 84:2582-2594.

Eyre JA, Miller S, Clowry GJ, Conway EA, Watts C (2000) Functional corticospinal projections are established prenatally in the human foetus permitting involvement in the development of spinal motor centres. Brain 123:51-64.

Eyre JA, Taylor JP, Villagra F, Smith M, Miller S (2001) Evidence of activitydependent withdrawal of corticospinal projections during human development. Neurology 57:1543-1554.

Friel K, Martin JH (2003) Activity-dependent competition between developing axons after sensory-motor cortex inactivation drives the topographic organization of the cat corticospinal system. Soc Neurosci Abstr 29:676.13

Fritz N, Illert M, Saggau P (1986a) Location of motoneurones projecting to the cat distal forelimb. I. deep radial motornuclei. J Comp Neurol 244:286-301.

Fritz N, Illert M, Reeh P (1986b) Location of motoneurons projecting to the cat distal forelimb. II. Median and ulnar motornuclei. J Comp Neurol 244:302-312.

Galea MP, Darian-Smith I (1995) Postnatal maturation of the direct corticospinal projections in the macaque monkey. Cereb Cortex 5:518-540.

Ghez C, Gordon J, Ghilardi M-F, Christokos CN, Cooper SE (1990) Roles of proprioceptive input in the programming of arm trajectories. Cold Spring Harbor Symp Quant Biol 55:837-847.

Goodman CS, Shatz CJ (1993) Developmental mechanisms that generate precise patterns of neuronal connectivity. Cell [Suppl] 10:77-98.

Gribnau AA, de Kort EJ, Dederen PJ, Nieuwenhuys R (1986) On the development of the pyramidal tract in the rat. II. An anterograde tracer study of the outgrowth of the corticospinal fibers. Anat Embryol (Berl) 175:101-110.

Hein A (1974) Prerequisite for development of visually guided reaching in the kitten. Brain Res 71:259-263.

Held R, Bauer JA (1967) Visually guided reaching in infant monkeys after restricted rearing. Science 155:718-720.

Hofsten CvP (1982) Eye-hand coordination in the newborn. Dev Psychol 3:450-461.

Hongo T, Kitazawa S, Ohki Y, Xi M-C (1989) Functional identification of last-order interneurones of skin reflex pathways in the cat forelimb segments. Brain Res 505:167-170.

Huttenlocher PR, Bonnier C (1991) Effects of changes in the periphery on development of the corticospinal motor system in the rat. Brain Res Dev Brain Res 60:253-260.

Inglis FM, Zuckerman KE, Kalb RG (2000) Experience-dependent development of spinal motor neurons. Neuron 26:299-305.

Jankovic J (1994) Botulinum toxin in movement disorders. Curr Opin Neurol 7:358-366.

Jootsten E, Gribnau A, Dederen P (1987) An anterograde tracer study of the developing corticospinal tract in the rat: three components. Brain Res Dev Brain Res 36:121-130.

Kuypers HGJM (1962) Corticospinal connections: postnatal development in the rhesus monkey. Science 138:676-678.

Levine MS, Hull CD, Buchwald NA (1980) Development of motor activity in kittens. Dev Psychobiol 13:357-371.

Li Q, Martin JH (2000) Postnatal development of differential projections from the caudal and rostral motor cortex subregions. Exp Brain Res 134:187-198.

Li Q, Martin JH (2001) Postnatal development of corticospinal axon terminal morphology in the cat. J Comp Neurol 435:127-141.

Li Q, Martin JH (2002) Postnatal development of connectional specificity of corticospinal terminals in the cat. J Comp Neurol 447:57-71.

Martin GF, Cabana T, Culberson JL, Curry JJ, Tschismadia I (1980) The early development of corticobulbar and corticospinal systems: studies using the North American opossum. Anat Embryol 161:197-213.

Martin JH, Ghez C (1993) Differential impairments in reaching and grasping produced by local inactivation within the forelimb representation of the motor cortex in the cat. Exp Brain Res 94:429-443.

Martin JH, Lee S (1999) Activity-dependent competition between developing corticospinal terminations. NeuroReport 10:2277-2282.

Martin JH, Cooper SC, Ghez C (1995) Kinematic analysis of reaching in the cat. Exp Brain Res 102:379-392.

Martin JH, Kably B, Hacking A (1999) Activity-dependent development of cortical axon terminations in the spinal cord and brain stem. Exp Brain Res 125:184-199.

Matsushita M, Hosoya Y, Ikeda M (1979) Anatomical organization of the spinocerebellar system in the cat, as studied by retrograde transport of horseradish peroxidase. J Comp Neurol 184:81-106.

Misiaszek JE, Pearson KG (2002) Adaptive changes in locomotor activity following botulinum toxin injection in ankle extensor muscles of cats. J Neurophysiol 87:229-239.

Ozaki S, Snider WD (1997) Initial trajectories of sensory axons toward laminar targets in the developing mouse spinal cord. J Comp Neurol 380:215-229.

Porter R, Lemon R (1993) Corticospinal function and voluntary movement. Oxford: Oxford Science.

Pullman SL, Green P, Fahn S, Pedersen SF (1996) Approach to the treatment of limb disorders with botulinum toxin A. Arch Neurol 53:617-624.

Sakamoto T, Arissian K, Asanuma H (1989) Functional role of sensory cortex in learning motor skills in cats. Brain Res 503:258-264.

Satomi H, Takahashi K, Aoki M, Kosaka I (1988) Anatomical evidence for the re-crossing of lateral corticospinal fibers via the posterior gray commissure in the cat spinal cord. Neurosci Lett 88:157-160.

Schiavo G, Matteoli M, Montecucco C (2000) Neurotoxins affecting neuroexocytosis. Physiol Rev 80:717-766. 
Simpson DM (2000) Treatment of spasticity with botulinum toxin. Muscle Nerve 23:447-449.

Solomons G, Solomons HC (1975) Motor development in Yucatecan infants. Dev Med Child Neurol 17:41-46.

Stanfield BB (1992) The development of the corticospinal projection. Prog Neurobiol 38:169-202.

Sterling P, Kuypers HG (1967) Anatomical organization of the brachial spinal cord of the cat. II. The motoneuron plexus. Brain Res 4:16-32.

Super CM (1976) Environmental effects on motor development: the case of African infant precocity. Dev Med Child Neurol 18:561-567.

Theriault E, Tatton WG (1989) Postnatal redistribution of pericruciate motor cortical projections within the kitten spinal cord. Brain Res Dev Brain Res 45:219-237.

Tresch MC, Saltiel P, Bizzi E (1999) The construction of movement by the spinal cord. Nat Neurosci 2:162-167. van der Meer AL, van der Weel FR, Lee DN (1995) The functional significance of arm movements in neonates. Science 267:693-695.

Villablanca JR, Olmstead CE (1979) Neurological development of kittens. Dev Psychol 12:101-127.

Walton K (1998) Postnatal development under conditions of simulated weightlessness and space flight. Brain Res Brain Res Rev 28:25-34.

Westerga J, Gramsbergen A (1993) Development of locomotion in the rat: the significance of early movements. Early Hum Dev 34: $89-100$.

Willis JK, Morello A, Davie A, Rice JC, Bennett JT (2002) Forced use treatment of childhood hemiparesis. Pediatrics 110:94-96.

Wise SP, Hendry SHC, Jones EG (1977) Prenatal development of sensorimotor cortical projections in cats. Brain Res 138:538-544.

Zelazo PR, Selazo NA, Kolb S (1972) Walking in the newborn. Science 176: $314-315$. 